\section{Avaliação continuada da educação permanente na atenção à criança na estratégia saúde da família}

\section{Continuous evaluation of permanent education concerning child care as part of the family health strategy}

Katia Virginia de Oliveira Feliciano 1

Maria Helena Kovacs2

Inês Eugênia Ribeiro da Costa 3

Maria da Graça Oliveira 4

Ana Maria Siqueira Araújo 5

\section{Abstract}

This article presents a continuous evaluation of work involving permanent education regarding childcare and the results that can be attributed to it, as carried out by family health teams managed jointly by the Community Outreach Program of the Instituto Materno Infantil Prof. Fernando Figueira, IMIP and the Municipal Departments of Health of the city Recife and the city of Olinda, State of Pernambuco, Brazil. With the support of external consultants, the group carried out systematic critical reflection, organized around the categories of performance, effects and effectiveness, with a view to enhancing the work under way. The sources of information used were the daily registers of the group and the teams and their views regarding the work. Despite the difficulty of transforming the changes brought about into routines, continuous education seems to have improved the quality of childcare. The satisfaction the teams feel corroborates this feeling that this work is both feasible and productive.

Key words Child health (Public Health), Family Health Program, Program evaluation

\section{Resumo}

Este artigo apresenta a avaliação continuada do trabalho realizado e os resultados que podem ser atribuídos à educação permanente na atenção à criança, desenvolvida junto às equipes de saúde da família, com gerência compartilhada pelo Programa de Extensão Comunitária do Instituto Materno Infantil Prof. Fernando Figueira (IMIP), e Secretarias Municipais de Saúde do Recife e Olinda, em Pernambuco, Brasil. Com apoio de consultores externos, o grupo executor realizou reflexão crítica sistemática, orientada pelas categorias desempenho, efeitos e efetividade, visando aprimorar o trabalho em seu decorrer. As fontes de informação foram os registros diários do grupo e das equipes e as percepções sobre o trabalho. Apesar da dificuldade de transformar em rotina as mudanças alcançadas, a educação permanente tem qualificado a atenção à criança. A satisfação das equipes reforça o sentimento de sua fecundidade e operacionalidade.

Palavras-chave Saúde da criança, Programa Saúde da Família, Avaliação de Programas e Projetos de Saúde 


\section{Introdução}

A estratégia saúde da família se diferencia, entre outros aspectos, porque pretende assegurar a eqüidade do acesso aos cuidados de saúde para atender às necessidades individuais, familiares e comunitárias; compromete-se a organizar os serviços, tendo a integralidade como eixo condutor das práticas; propõe-se a prestar assistência resolutiva na promoção, prevenção e recuperação em saúde dos distintos segmentos populacionais; redefine as atribuições dos membros da equipe, competindo ao médico cuidar de toda a família, à parte o sexo e a idade; e confere particular importância ao monitoramento das ações por meio do Sistema de Informação de Atenção Básica (SIAB).1,2 Nesse modelo assistencial, a atenção à criança resulta de trabalhos interdependentes e complementares, onde a clínica emerge como espaço para apreensão mais global das necessidades de saúde individuais, pressupondo a existência de profissionais generalistas.

Porém, no cotidiano das equipes, o médico que cuida da criança não é generalista e pode não ser pediatra, sendo também, geralmente, insuficiente a capacitação de enfermeiros e agentes comunitários de saúde para cumprir suas atribuições na assistência básica e ações de vigilância epidemiológica e sanitária à criança. Logo, para que ocorram as mudanças requeridas pela saúde da família se faz fundamental a educação permanente que, de acordo com Ministério da Saúde (2000: 11)3 foi:

[...] concebida dentro das premissas básicas de ser um processo constante de promoção e desenvolvimento integral e contextualizado da equipe, centrando-se nas circunstâncias e problemas de seu processo de trabalho, de modo crítico e criativo.

Assim, na construção do poder resolutivo frente às necessidades de saúde presentes em uma determinada realidade é dada primazia à reflexão crítica, à capacidade técnica e às relações humanas.

A resolutividade manifesta a aptidão para solucionar o problema de saúde da pessoa, pressupondo a integralidade do cuidado que resulta, na atenção básica, da capacidade de atender às necessidades de saúde mediante atuação direta da equipe, encaminhamento para outros níveis de densidade tecnológica, articulação dos recursos da comunidade e fornecimento de informações aos níveis organizacionais com poder de articulação intersetorial. Cabe ressaltar que a operacionalização adequada da saúde da família fica ainda mais difícil porque são exigidas mudanças profundas nas concepções e práticas habituais daqueles que, nos distintos contextos locais, estão envolvidos no seu processo de implantação. ${ }^{2,4-7} \mathrm{Na}$ opinião de $\mathrm{Ceccim}^{8} \mathrm{o}$ que distingue a educação permanente em saúde é, justamente, sua porosidade à realidade dos contextos, das ações e dos serviços de saúde.

No âmbito da saúde da criança, desde 1996, o Ministério da Saúde vem treinando médicos e enfermeiros na Atenção Integrada às Doenças Prevalentes da Infância (AIDPI) para integrar no atendimento clínico as ações curativas, preventivas e promocionais. ${ }^{9}$ Todavia, permanecia a preocupação com o desempenho dos agentes, devido à importância das atividades domiciliares nessa fase do ciclo de vida. No ano 2000, o Ministério da Saúde firmou convênio com o Instituto Materno Infantil Prof. Fernando Figueira (IMIP) para que os profissionais do Programa de Extensão Comunitária (PEC), juntamente com consultores e técnicos do Ministério adaptassem o material teórico da Atenção Integral as Doenças Prevalentes na Infância (AIDPI) para o agente de saúde. 10 No início de 2002 foram distribuídos, nacionalmente, os documentos "Atenção Básica à Saúde da Criança - texto de apoio para o Agente Comunitário de Saúde"11 e "Manual de condutas para Agentes Comunitários de Saúde - Atenção Integrada às Doenças Prevalentes na Infância".12

O PEC, em 2001, guiando-se pelos princípios e diretrizes da estratégia saúde da família, ${ }^{2}$ reorienta o modelo de assistência à saúde da criança e da mulher que implementava, desde 1983, em três unidades de saúde. Em parceria com as Secretarias Municipais de Saúde do Recife e de Olinda, passa a compartilhar com os Distritos Sanitários a supervisão técnica e administrativa (manutenção de equipamentos e estrutura física) de 12 unidades de saúde da família, onde atuam 19 equipes. Diante das limitações observadas na assistência prestada à criança nessas unidades e da falta de perspectiva, na época, quanto à incorporação pelas Secretarias de Saúde, em curto prazo, de novos elementos a sua prática de capacitação de quadros institucionais, foi iniciado processo de preceptoria como meio de desenvolvimento da educação continuada.

A preceptoria na atenção à saúde da criança tinha como objetivos ampliar o conhecimento do perfil de morbidade local e promover o aperfeiçoamento de médicos e enfermeiras para aumentar o poder resolutivo da equipe. A atividade consistia na discussão de casos clínicos, selecionados pelos profissionais das equipes, previamente agendados com três médicas pediatras (preceptores). Afora as inadequações da abordagem clínica, essas discussões permitiram identificar a omissão de registro no prontuário, 
a vacinação incompleta para a idade, o abandono precoce do aleitamento materno, as deficiências do acompanhamento do crescimento, o despreparo do agente comunitário e a falta de articulação no interior da equipe. Como as dificuldades da atenção à saúde da criança não se restringiam à prática clínica, surgiram inquietações acerca da maneira de pensar $\mathrm{e}$ de fazer a educação em serviço.

Com o aporte de profissionais da área da avaliação, a partir de abril de 2002, foi aprofundada a reflexão crítica sobre "o quê", o "como" e o "porquê" do trabalho que vinha sendo efetuado, o que propiciou o reconhecimento de que se havia subestimado a magnitude dos desafios envolvidos na transição entre modelos assistenciais tão diferenciados. Desafios que incluíram também a mudança de concepção dos preceptores sobre a integralidade, que até então era tratada como uma dimensão da prática médica, relacionada a uma atitude médica desejável, característica da boa medicina. Essa ampliação de horizontes deixou clara a necessidade de uma educação permanente com objetivos e estratégias focalizados na capacitação e apoio organizacional para favorecer a articulação dos elementos preventivos, curativos e promocionais como parte do processo de trabalho de uma equipe multiprofissional na saúde da família.

Para tanto, o modelo de preceptoria foi transformado na atuação em um grupo de supervisão que exerce suas atividades como um instrumento de desenvolvimento das equipes e estratégia gerencial para organização do trabalho. Desde então, está prevista para cada equipe visita quinzenal de pediatra para efetuar atividades de apoio à puericultura, ao diagnóstico e tratamento de enfermidades, dando ênfase à aplicação da AIDPI, mediante a discussão de casos clínicos, a oferta de cursos e o suporte para realização da programação, execução e monitoramento das ações. A necessidade de ter um olhar mais amplo para a capacitação dos profisssionais da saúde da família 4 e as dificuldades para a constituição de equipes de referência multiprofissionais, ${ }^{13}$ aumentam de modo considerável a importância de utilização desta estratégia de educação permanente em contextos aproximados.

A avaliação continuada da implantação deste projeto foi estruturada com base no processo de planejamento, de modo a tornar possível o máximo aproveitamento das informações produzidas. O monitoramento, cujo sentido advém de seu compromisso com a discussão e a reformulação periódica do trabalho, é um instrumento básico da avaliação processual.14 Apesar de restrito aos aspectos nucleares do trabalho planejado, ou seja, ao "que é feito" por referência ao que "se pode e/ou se deve fazer", solicita o debate reflexivo sobre as práticas e os contextos onde as mesmas estão inseridas. Em uma conjuntura em que o Ministério da Saúde, por intermédio do Departamento de Atenção Básica da Secretaria de Atenção à Saúde, prioriza a institucionalização da avaliação, 15 este artigo apresenta aspectos do processo sistemático de avaliação da educação permanente relacionados ao trabalho realizado e aos resultados que lhe podem ser atribuídos.

\section{Métodos}

Trata-se da avaliação continuada da educação permanente no âmbito da atenção à saúde da criança efetuada junto às 19 equipes de saúde da família (17 da Secretaria de Saúde do Recife e duas de Olinda) vinculadas ao PEC, referente ao período compreendido entre abril de 2002 e dezembro de 2005, em que foi utilizada abordagem voltada para o desenvolvimento pelo grupo executor da reflexão sobre sua própria prática. É inegável que a participação direta dos envolvidos na ação aumenta a capacidade da avaliação para melhorar o trabalho durante o seu decorrer, mas algumas desvantagens são atribuídas ao avaliador interno, como fazer parte da estrutura de poder e talvez ter dificuldade para expressar juízos de valor. 16

Por isso, as reuniões semanais de avaliação do grupo contaram, durante os primeiros meses, com a participação semanal ou quinzenal de dois consultores externos ao grupo, de formações e experiências diferenciadas, periodicidade que posteriormente ficou sendo trimestral. Desse modo, pretendia-se superar eventuais dificuldades para abordar os desconfortos experimentados no cotidiano e para questionar os conceitos e as práticas vigentes no grupo e nas equipes, estimulando uma visão cooperativa por meio de um processo coletivo de análise e reflexão. Esta perspectiva está comprometida com o desenvolvimento da consciência crítica para a tomada de decisões e da capacidade de intervenção sobre a realidade, elementos encontrados nos enfoques que visam à aprendizagem social e organizacional, logo, elementos que formalizam a avaliação como um dispositivo de mudança. ${ }^{17-19}$

Reconhecendo a especificidade e a interdependência da educação permanente e da assistência à saúde da criança, a avaliação contemplou a definição do que precisava ser feito mediante a educação permanente, a problematização do contexto de sua realização, o processo de trabalho do grupo e os seus resultados, intercalando aspectos teóricos, e o delineamento das mudanças necessárias no curso do 
trabalho educativo. Esta abordagem, portanto, utilizou a Teoria de Programa desenvolvida por Chen, em 1990, citado por Worthen et al., 16 como ferramenta para compreender a operacionalização da proposta de atuação e orientar a avaliação.

Os distintos significados implícitos na noção de resultados foram desdobrados em três categorias inter-relacionadas. Recorreu-se a Draibe ${ }^{19}$ para demarcar o "desempenho" como o cumprimento das metas propostas pelo grupo e os "efeitos" como a aprendizagem das instituições parceiras e do grupo que implementa as ações, bem como a Pabón ${ }^{20}$ para definir a "efetividade" como as mudanças alcançadas nas características da atenção à saúde da criança que se pretendia transformar. A efetividade foi avaliada por meio de uma comparação "antes - depois", na qual maio de 2003 (começo da programação local) foi considerado o ponto inicial, sendo utilizados os dados do Sistema de Informação da Atenção Básica (SIAB) e as notas sistemáticas sobre o trabalho cotidiano.

As fontes de informação foram as notas individuais sobre o dia-a-dia do trabalho junto às equipes: os aspectos positivos e as dificuldades encontradas na operacionalização das atividades previstas e as repercussões da prática educativa na atuação das equipes (normas, rotinas, articulação entre atividades, planejamento e monitoramento das ações); o questionário de manejo de casos clínicos; os relatórios dos cursos ministrados e as impressões acerca das vivências do grupo. Também foram utilizadas as informações produzidas pelas equipes por intermédio dos prontuários, dos instrumentos de programação local, do SIAB, além das percepções de seus integrantes sobre o trabalho realizado pelo grupo.

Por meio do percurso reflexivo e compreensivo da "dialética de pergunta e resposta", de acordo com o movimento hermenêutico proposto por Gadamer, ${ }^{21}$ os consultores externos introduziam temas e informações, no sentido de possibilitar respostas para perguntas como: A educação permanente está cumprindo os objetivos propostos? Quais são os fatores que têm facilitado e dificultado a operacionalização da educação permanente? Ocorreram correções de processos e procedimentos, como resultado do monitoramento do trabalho realizado pelo grupo? São constatadas melhoras nas capacidades profissionais das equipes, como resultado da educação permanente? As mudanças alcançadas passaram a ser parte das atividades de rotina das equipes? Os beneficiários estão satisfeitos? Além disso, interroga: Há possibilidade de transferir este modo de atuação para outros contextos?

É conveniente se atentar, por um lado, para o rigor que perpassou a produção e a análise contextualizada das informações em que foram utilizados, além dos preceitos técnicos e estratégicos para a concretização de procedimentos e atividades que comumente servem de referência à avaliação normativa, os pressupostos e valores dos participantes das ações. 17,18 Por outro lado, para o caráter reflexivo adotado no que pode se definir como uma descrição expandida desta experiência, motivando a opção que foi feita, neste artigo, pela forma de narrativa para comunicar os aspectos considerados mais significativos da avaliação do processo de educação permanente.

\section{Resultados}

\section{O desempenho e os efeitos da educação permanente}

Em 2002, todos os 19 médicos e 19 enfermeiros, 108 agentes e 19 auxiliares de enfermagem foram treinados na AIDPI. No caso de um desses ser substituído por profissional que não foi treinado, se garante a capacitação. Houve, no período estudado, substituição de médicos e enfermeiros em, respectivamente, 12 e 7 equipes. As pediatras realizaram o acompanhamento de visitas de cada agente comunitário para fortalecer a aplicação do "Manual de condutas para Agentes Comunitários de Saúde Atenção Integrada às Doenças Prevalentes na Infância", 12 tendo feito um maior número de visitas com aqueles que demandavam maior suporte técnico. Ao mesmo tempo, apoiavam o processo de supervisão dos enfermeiros nas equipes em que esses profissionais estiveram presentes em todas as etapas do treinamento dos agentes.

A discussão de casos clínicos que atende a solicitação profissional tem contado, em geral, com a participação do médico, do enfermeiro e do agente da microárea de residência da criança, utilizando instrumento próprio para monitorar a qualidade da assistência. Por sua vez, a partir do observado na rotina das equipes, as auxiliares de enfermagem receberam um reforço na capacitação para que pudessem se integrar de fato ao trabalho de promoção e prevenção executado pelos agentes junto às famílias e proceder à identificação dos sinais de alerta e de perigo das doenças prevalentes na infância. Buscou-se também melhorar a qualidade das atividades de imunização (conservação de vacinas, esquema de vacinação e contra-indicações) e avaliação do crescimento (manutenção da balança, aferição, registro e interpretação do peso). 
Entre maio de 2002 e abril de 2003, médicos, enfermeiros, cirurgiões-dentistas e agentes comunitários das 19 equipes de saúde, bem como representantes dos Distritos Sanitários onde essas estão localizadas, foram capacitados, por profissionais de fora do PEC, para proceder à coleta, ao processamento, à análise e à interpretação de dados do SIAB. Foi feito trabalho adicional com o grupo de educação permanente sobre a programação como eixo organizador da utilização desses dados. Em maio de 2003, com a anuência das Secretarias de Saúde do Recife e Olinda, o grupo iniciou um processo tentativo de programação local: a) elaboração de formulários para definir as necessidades de saúde, as prioridades, a capacidade potencial da equipe e as metas a serem alcançadas e b) negociação com as equipes para escolher os parâmetros aplicados e efetuação da programação das atividades a serem executadas pelas equipes.

No início, o grupo de educação permanente realizava reuniões semestrais com cada uma das equipes para avaliar as ações, identificar as dificuldades e tentar a introdução de melhorias na execução do trabalho. Apesar dos avanços alcançados persistia a desvalorização do sistema informação como ferramenta de planejamento, as deficiências na coleta e supervisão da produção de dados. Por isso, foi adotada a tática de reforçar a avaliação do trabalho pelo próprio agente. Desde outubro de 2004 são efetuadas reuniões mensais em que os agentes comunitários, com apoio dos demais integrantes das equipes, calculam e interpretam indicadores selecionados, avaliam a qualidade dos dados produzidos, refletem sobre a operacionalização das atividades e resultados obtidos e redefinem prioridades e modos de atuação.

As articulações com outros setores do IMIP resultaram na participação dos médicos e enfermeiras nos cursos de atualização em aleitamento materno, prevenção da violência doméstica e de diagnóstico precoce do câncer infantil. Em 2005, para suprir uma das limitações da AIDPI, as pediatras treinaram médicos, enfermeiras e agentes, para realizar a avaliação clínica do desenvolvimento infantil. Todo o grupo do PEC preparou os componentes das equipes, bem como profissionais indicados pelos Distritos Sanitários, para atuar na atenção integrada à família, como parte da parceria com o Fundo das Nações Unidas para a Infância (UNICEF) no projeto Família Brasileira Fortalecida.

As características das relações entre o IMIP e os Distritos Sanitários (três da Secretaria de Saúde do Recife e um de Olinda) traduzem a especificidade do processo de gerência, podendo tornar mais ou menos árduas as negociações para a efetivação da educação permanente (substituição de profissionais, cronograma de cursos, adequação de parâmetros, etc). Entretanto, as decisões tomadas têm criado condições para a continuidade e ampliação da experiência. A sistematicidade da aproximação à prática cotidiana das equipes tem favorecido uma identificação mais rápida das necessidades e o caráter contínuo da avaliação do processo educativo tem permitido, em determinadas circunstâncias, uma maior presteza nas respostas.

O grupo, que quando foi criado era constituído por três médicas pediatras, passou a ser formado, em 2002, por duas pediatras, uma fonoaudióloga com atuação no apoio organizacional e uma nutricionista responsável pela coordenação do PEC. Desde o início de 2004 o grupo conta com as duas pediatras e uma sanitarista que coordena as atividades de extensão. Cada pediatra responde por seis unidades (9 e 10 equipes) e dedica 20 horas semanais ao processo educativo, enquanto a sanitarista tem carga horária de 40 horas. No momento em que uma equipe demanda mais de uma visita quinzenal, aquelas cujo trabalho apresenta maiores condições de sustentação têm o intervalo da visita aumentado. Há descontinuidade da ida à unidade quando cursos estão sendo ministrados.

A redução dos integrantes do grupo ocorreu justamente no período em que estavam sendo intensificadas as atividades de programação e monitoramento. $\mathrm{O}$ número insuficiente de profissionais e a superposição de tarefas que demandam adaptações nesta rotina são condicionantes negativos do desempenho da educação permanente. Todavia, os membros do grupo constataram o fortalecimento de suas capacidades profissionais no que diz respeito à compreensão das necessidades da organização e do processo de trabalho voltado para a operacionalização da integralidade da atenção à criança na saúde da família, além da maior familiaridade com a avaliação, que tem propiciado um conhecimento mais aprofundado sobre as práticas e seus contextos.

\section{A efetividade da educação permanente}

Apesar das enormes variações observadas no desempenho das equipes, indo desde a cobertura total até o seguimento de apenas uma terça parte das crianças a serem atendidas, houve um crescimento importante da cobertura de atendimento de puericultura em menores de dois anos. Nas equipes com baixa cobertura é comum que os enfermeiros reclamem da sobrecarga de trabalho, justificando que falta tempo para realizar o acompanhamento da 
criança sadia devido à superposição de atividades. $\mathrm{O}$ excesso de atribuições do enfermeiro é um fato, mas o que sobressai nessas equipes é a falta de articulação técnica e as dificuldades de interação comunicativa entre os profissionais, ainda que não sejam incomuns os acordos para acomodar outros interesses.

Embora o incêndio ocorrido em janeiro de 2005 na Diretoria de Pesquisa do IMIP tenha destruído os dados do PEC, o grupo reconhece a melhoria da qualidade no manejo dos casos de doença. Em 2005, dos 121 casos avaliados $81,8 \%$ tinham anamnese satisfatória, $76 \%$, exame físico apropriado, 73,6\% contexto familiar discutido e $67,8 \%$ hipótese diagnóstica aceita pelas pediatras. Por outro lado, em $13,2 \%$ a conduta foi incorreta (droga escolhida e orientação alimentar); exames complementares necessários não foram solicitados em $42,2 \%$ dos casos, não havia indicação para $13,2 \%$ das referências, enquanto $12,4 \%$ não foram encaminhados apesar de necessitarem. Houve equívoco na escolha de unidades de referência e no tipo de especialista indicado, sendo marcante a fragilidade das articulações formais na rede hierarquizada. Em $64,4 \%$ dos casos o suporte foi requerido pelos enfermeiros: $50,4 \%$ por conta própria e $14 \%$ com o médico. A AIDPI foi utilizada em $61,4 \%$ dos casos, com os enfermeiros respondendo pela sua aplicação em $89,5 \%$ desses.

Atualmente, os médicos de sete equipes são pediatras, mas à parte a especialidade entre esses profissionais, existe uma forte resistência para utilizarem a AIDPI. São principalmente os pediatras que contribuem para que enfermeiros, auxiliares e agentes desenvolvam uma melhor abordagem das doenças infantis de maior prevalência, reconhecendo a relevância da assistência oportuna, uma vez que se colocam disponíveis para esclarecer as dúvidas e aproveitam a consulta da criança para chamar a atenção sobre algum aspecto importante do caso. A solicitação de apoio exclusiva do enfermeiro (em algumas equipes apenas esse profissional pede que sejam discutidos casos clínicos) quase sempre é um indicativo de que falta articulação técnica ou até mesmo de que são delicadas as relações interpessoais com o médico.

Uma avaliação normativa da visita domiciliar do agente de saúde efetuada no final de 2002 constatou que $91,7 \%$ dos menores de dois meses tiveram os sinais de perigo e $83,3 \%$ os sinais de alerta avaliados corretamente, enquanto no segmento entre dois meses e cinco anos em $73,1 \%$ foi cumprida a padronização para verificação dos sinais de perigo e em $80,8 \%$ para os sinais de alerta. Um percentual correspondente a $99 \%$ dos menores de dois meses foi submetido à avaliação da situação vacinal, ao passo que as mães de, respectivamente, $96,9 \%$ e $80,4 \%$ foram adequadamente orientadas sobre o aleitamento materno e os cuidados com o bebê. 10

A visita do agente permaneceu sendo realizada com maior qualidade nas poucas equipes em que os enfermeiros, simultaneamente, aplicam a AIDPI, supervisionam a utilização rotineira do "Manual de Condutas" 12 pelos agentes e buscam o fortalecimento e a manutenção de um fluxo regular de encaminhamento interno. Já naquelas em que os enfermeiros estão distanciados do acompanhamento do agente e é frágil a conexão dos trabalhos parcelares, observa-se uma tendência para ocorrer uma utilização residual da AIDPI pelos agentes. Nessas circunstâncias, agentes, enfermeiros e auxiliares de enfermagem receberam treinamento teórico e prático adicional. Em 2004 foi feita uma outra avaliação normativa da visita dos agentes, a qual mostrou comportamento similar ao de 2002, sendo realizada uma nova capacitação com esses componentes da equipe.

No âmbito da atenção à criança, com os parâmetros ajustados com base nas padronizações estabelecidas pelo Ministério da Saúde,22 a programação local tem possibilitado que algumas equipes desenvolvam um plano de trabalho que, paralelamente, amplia a cobertura e prioriza a concentração de atividades nos grupos mais vulneráveis. Nas equipes em que os membros se restringem a fazer o cronograma de suas atividades de maneira individualizada é evidente a falta de articulação técnica e de responsabilidade coletiva na busca de solução para os problemas identificados no âmbito da atenção à saúde da criança. Essa cisão entre as parcelas que compõem a totalidade das práticas pode ser reforçada também pela rotatividade de médicos e enfermeiras.

Naquelas equipes em que existe um maior reconhecimento da interdependência da autonomia profissional é corriqueira a participação sistemática de todos os componentes na reunião mensal do monitoramento. Isso tem permitido uma reflexão crítica sobre o papel do agente na comunidade, evidenciado a importância da integração das atividades; mantidos os avanços na padronização e registro de dados do SIAB relativos à família e à criança; despertado maior compreensão da necessidade de programação e monitoramento para resolutividade do cuidado; propiciado a identificação de microáreas e famílias mais vulneráveis. Nas demais, é descontínua a participação nas reuniões, sobretudo, dos médicos, são comuns as variações na qualidade da coleta dos 
dados e maiores as dificuldades para reorientar as ações.

Os beneficiários da educação permanente na atenção à criança têm manifestado satisfação porque contam com apoio no esclarecimento de dúvidas, no encaminhamento de casos e na manutenção de equipamentos e área física. Contudo, alguns externam o sentimento de que são mais exigidos em comparação com os componentes das equipes que não participam deste convênio e, particularmente, os agentes comunitários demandam um maior conhecimento do grupo de educação permanente sobre a realidade local, tendo em vista a influência dos modos de pensar e agir que prevalecem na comunidade sobre os resultados esperados.

\section{Discussão}

Em relação ao cotidiano do grupo é preocupante o número de equipes que ficam sob a responsabilidade de cada pediatra, já que a educação permanente pressupõe trabalho em profundidade, logo, potencial para atendimento de necessidades particulares dos integrantes da equipe, sendo longo o tempo necessário para o processo de capacitação técnica e as transformações de concepções e práticas na atenção à criança. ${ }^{8}$ No entanto, apesar do número insuficiente de profissionais, foram feitas adaptações na programação de atividades no sentido de, paralelamente, fornecer suporte mais intensivo às equipes que apresentavam maior oscilação na qualidade da assistência prestada e realizar os cursos previstos, visando ao cumprimento dos objetivos traçados.

É inegável a efetividade da educação permanente, que tem se revelado essencial para reduzir a vulnerabilidade da população infantil. Nas unidades com gerência compartilhada pelo IMIP, os avanços na atenção à criança estão relacionados à correta utilização da estratégia de AIDPI por enfermeiros e agentes comunitários de saúde, em um cenário de demanda reprimida para atendimento médico. Sobretudo nas situações de trabalho em que se busca uma maior articulação das atividades, sendo sistemática a troca de informações entre os membros da equipe. Há de convir que a autonomia técnica que tem caracterizado, historicamente, o exercício da medicina torna mais crítica a integração da assistência médica como instrumento de um trabalho coletivo. 23

Semelhante ao constatado em pesquisas efetuadas por Pedrosa e Teles, ${ }^{6}$ e Silva e Trad, ${ }^{7}$ são muitas as dificuldades de interação na equipe de saúde da família, seja entre os profissionais de nível superior, seja entre esses profissionais e os agentes comu- nitários. Porém, à medida que o processo educativo cria condições para que a equipe se reúna e discuta, incentivando o debate reflexivo sobre as práticas e seus contextos, algumas equipes têm mostrado uma maior compreensão da especificidade e complementaridade dos papéis desempenhados pelos membros. 5,7,23 Isso amplia a percepção das necessidades de aprendizagem e organização, elemento imprescindível para o delineamento das alterações necessárias no curso da formação para o trabalho.

Um aspecto significativo da educação permanente desenvolvida pelo PEC tem sido justamente a tentativa de alimentar o processo de ação-reflexãoação mediante a participação de todos os membros da equipe nas definições relacionadas com a programação local e o monitoramento de sua execução. Busca-se assim que cada profissional reconheça a necessidade do trabalho do outro e da divisão de tarefas para operacionalizar a integralidade da atenção em saúde, superando o caráter fragmentário que perpassa a justaposição alienada de trabalhos. A qualidade do preenchimento dos instrumentos do SIAB passa a ser uma necessidade interna da equipe e a avaliação não se restringe aos indicadores de produtividade. $\mathrm{O}$ aprimoramento do senso crítico repercute sobre a capacidade de resolução dos problemas de saúde infantis presentes na realidade local, bem como fortalece o compromisso com os resultados esperados.

O modo operatório da educação permanente está de acordo com a prioridade conferida pelo Ministério da Saúde à institucionalização da avaliação, isto é, com a pretensão de criar um ambiente organizacional favorável à incorporação do processo avaliativo à rotina dos serviços básicos de saúde. 15 No entanto, considerando o papel assumido pelo planejamento local nas equipes vinculadas ao PEC, duas limitações merecem ser explicitadas. Primeiro, a integralidade da atenção compreendida no espaço macrossocial implica que a programação não se atenha ao setor saúde, ${ }^{24}$ mas essa ainda permanece restrita aos limites setoriais. Segundo, embora a legitimidade das decisões acerca de prioridades e objetivos remeta ao estabelecimento de pactos com a comunidade, este projeto de intervenção local ressalta o pólo técnico de atuação da equipe. $\mathrm{O}$ aperfeiçoamento deste processo pressupõe o aprofundamento da discussão com a comunidade sobre o possível de realizar, bem como a articulação com outras instituições de dentro e de fora do setor saúde.

Da mesma forma, a avaliação processual tem sido de valor inestimável para a qualificação do processo de educação permanente. A reflexão sistemática desenvolvida pelo grupo sobre os 
elementos constituintes da organização e da execução do trabalho vem alimentando a transformação de conceitos e práticas. Isso repercute de maneira bastante positiva sobre a capacidade de se manter atento às necessidades presentes nas distintas realidades onde se dão as vivências profissionais das equipes de saúde da família.3,8 Esse aspecto que adquire ainda maior significação quando se concorda

\section{Referências}

1. Brasil. Ministério da Saúde. Secretaria de Assistência à Saúde. Coordenação de Saúde da Família. Saúde da família: uma estratégia para reorientação do modelo assistencial. Brasília, DF; 1997.

2. Souza M, Costa Neto MM. A implantação da Unidade da Saúde da Família. Brasília, DF: Departamento de Atenção Básica, Secretaria de Políticas de Saúde do Ministério da Saúde; 2000. (Cadernos de Atenção Básica 1).

3. Brasil. Ministério da Saúde. Secretaria de Políticas de Saúde. Departamento de Atenção Básica. Educação permanente. Brasília, DF; 2000. (Cadernos de Atenção Básica. Programa de Saúde da Família, 3).

4. Rosa WAG, Labate RC. Programa Saúde da Família: a construção de um novo modelo de assistência. Revista LatAm Enfermagem. 2005; 13: 1027-34.

5. Ribeiro EM, Pires D, Blank VLG. A teorização sobre processo de trabalho em saúde como instrumental para análise do trabalho no Programa Saúde da Família. Cad Saúde Pública. 2004; 20: 438-46.

6. Pedrosa JIS, Teles JBM. Consenso e diferenças em equipes do Programa Saúde da Família. Rev Saúde Pública. 2001; 35: 303-11.

7. Silva IZQJ, Trad LAB. O trabalho em equipe no PSF: investigando a articulação técnica e a interação entre os profissionais. Interface: Com Saúde Educ. 2004; 9: 25-38.

8. Ceccim RB. Educação permanente em saúde: desafio ambicioso e necessário. Interface: Com Saúde Edu. 2004; 9 : 161-77.

9. Benguigui Y. Perspectivas en el control de enfermedades en los niños: Atención Integrada a las Enfermedades Prevalentes de la Infancia. Rev Bras Saúde Matern Infant. 2001; 1: 7-19.

10. Vidal SA, Silva EV, Oliveira MG, Siqueira AM, Felisberto E, Samico I, Cavalcante MGS. Avaliação da aplicação da estratégia da Atenção Integrada às Doenças Prevalentes da Infância (AIDPI) por agentes comunitários de saúde. Rev Bras Saúde Matern Infant. 2003; 3: 205-13. com a afirmação de Mattos $^{25}$ de que, no momento atual, a integralidade deixa de contemplar apenas às diretrizes programáticas que deveriam guiar o processo de trabalho e passa a assimilar as necessidades de saúde que emergem no contexto local, portanto, necessidades não consideradas na organização anteriormente dada.

11. Brasil. Ministério da Saúde. Secretaria de Políticas de Saúde. Departamento de Atenção Básica, Instituto Materno Infantil de Pernambuco (IMIP). Atenção Básica à Saúde da Criança: texto de apoio para o Agente Comunitário de Saúde. Brasília, DF; 2001.

12. Brasil. Ministério da Saúde. Secretaria de Políticas de Saúde. Departamento de Atenção Básica. Instituto Materno Infantil de Pernambuco (IMIP). Manual de condutas para Agentes Comunitários de Saúde - Atenção Integrada às Doenças Prevalentes na Infância. Brasília, DF; 2001.

13. Carvalho SR, Campos GWS. Modelos de atenção à saúde: a organização de equipes de referência na rede básica da Secretaria Municipal de Saúde de Betim, Minas Gerais. Conferência Nacional de Saúde On-Line, 1999. Disponível em URL: http://datasus.gov.br/cns/cns.htm [2005 outubro 19].

14. Sala A, Nemes MIB, Cohen DD. A avaliação na prática programática. In: Schraiber LB, Mendes-Gonçalves RB, Nemes MIB, organizadores. Saúde do adulto, programas e ações na unidade básica. São Paulo: Hucitec; 1996. p. 17393.

15. Felisberto E. Monitoramento e avaliação na atenção básica: novos horizontes. Rev Bras Saúde Matern Infant. 2004; 4: 317-21.

16. Worthen BR, Sanders JR, Fitzpatrick JL. Clareza ao demandar uma avaliação e definir suas responsabilidades. In: Worthen BR, Sanders JR, Fitzpatrick JL. Avaliação de programas: concepções e práticas. São Paulo: Gente; 2004. p. 269-339.

17. Furtado JP. Um método construtivista para a avaliação em saúde. Cien Saúde Coletiva. 2001; 6: 165-81.

18. Feliciano KVO. A relação entre o avaliador e o objeto avaliado. Rev Bras Saúde Matern Infant. 2005; 5 (Supl.1): 583-92.

19. Draibe SM. Avaliação de implementação: esboço de uma metodologia de trabalho em políticas públicas. In: Barreira MCRN, Carvalho MCB, organizadores. Tendências e perspectivas na avaliação de políticas e programas sociais. São Paulo: Instituto de Estudos Especiais da Pontifícia Universidade Católica de São Paulo; 2001. p.13-42. 
20. Pabón H. Evaluación de servicios de salud. 2. ed. Cali (Colombia): Universidad del Valle; 1987.

21. Gadamer H-G. Verdade e método. 2. ed. Petrópolis: Vozes; 1998.

22. Brasil. Ministério da Saúde. Secretaria de Políticas de Saúde. Departamento de Atenção Básica. Parâmetros para programação das ações básicas de saúde. Brasília, DF; 2001.

23. Peduzzi M, Palma JJL. A equipe de saúde. In: Schraiber LB, Mendes-Gonçalves RB, Nemes MIB, organizadores. Saúde do adulto, programas e ações na unidade básica. São Paulo: Hucitec; 1996. p. 234-50.

Recebido em 10 de julho de 2006

Versão final apresentada em 6 de outubro de 2007

Aprovado em 17 de novembro de 2007
24. Rivera FJU. Planejamento e programação em saúde: um enfoque estratégico. São Paulo: Cortez; 1989.

25. Mattos RA. Os sentidos da integralidade: algumas reflexões acerca de valores que merecem ser definidos. In: Pinheiro R, Mattos RA, organizadores. Os sentidos da integralidade na atenção e no cuidado à saúde. Rio de Janeiro: Universidade Estadual do Rio de Janeiro, Instituto de Medicina Social, Associação Brasileira de Pós-Graduação em Saúde Coletiva; 2001. p. 39-64. 